\title{
PULSED HEATING EXPERIMENTS AT 34 GHZ*
}

\author{
O.A. Nezhevenko ${ }^{1}$, V.P. Yakovlev ${ }^{1}$, J.L. Hirshfield ${ }^{1,2}$, and G.V. Serdobintsev ${ }^{2, \#}$ \\ ${ }^{1}$ Omega-P, Inc., New Haven, CT 06511, USA, \\ ${ }^{2}$ Physics Department, Yale University, New Haven, CT 06520, USA
}

\begin{abstract}
Pulsed heating is one of the main factors that limit accelerating gradient in normal conducting linear accelerators. In this paper a special test device is described that is designed to determine the maximum achievable surface electric field limit due to pulsed heating and metal fatigue. The power source for driving the test device is to be the Omega-P $34 \mathrm{GHz}$ pulsed magnicon. Utilization of a few MW of $\mu$ sec pulse width should allow one to obtain pulsed heating excursions exceeding $600^{\circ} \mathrm{C}$. The test device is designed with a to allow measurements for copper, for other materials, and for different procedures of surface preparation.
\end{abstract}

\section{INTRODUCTION}

Two phenomena limit the practical utility of accelerating structures: $\mathrm{rf}$ breakdown that limits the acceleration gradient, and surface fatigue due to pulsed heating that limits the structure lifetime. \#

Pulsed heating [1] is caused by the eddy currents created by a high-power rf pulse. Mechanical stress appears in the thin surface layer of metal due to a temperature rise $\Delta T$. When the rise in temperature is above a "safe" value $\Delta T_{s}$, the mechanical stress grows large enough to create microscopic damages in the metal. The damage accumulates with each succeeding pulse and the cavity surface is destroyed after a certain number of pulses. In practice, the destruction of the metal surface occurs when the mechanical stress exceeds its elastic limit, which allows one to estimate a safe temperature threshold [1,2], namely $\Delta T_{s} \approx 2 \sigma / \alpha E$, where $\sigma$ is the yield stress, $\alpha$ is the coefficient of linear thermal expansion, and $E$ is the elastic modulus. For copper this estimate gives a safe threshold for temperature rise $\Delta T_{s}$ of about $110 \mathrm{C}^{\circ}$.

At present, pulse heating experiments using $11.424 \mathrm{GHz}$ cavities have been carried out at SLAC [3]. The experiments demonstrated that pulsed heating with a temperature rise of $120^{\circ} \mathrm{C}$ showed modification and damage of the copper surface after $5.5 \times 10^{7}$ pulses. It also showed that significant damage had occurred to the coupler aperture of the test cavity, where the temperature rise was about $250{ }^{\circ} \mathrm{C}$.

However, for practical needs it is important to know the temperature rise threshold and material life-time more exactly, because even at the moderately high gradients in the NLC design it is hard to reduce the local temperature rise to an acceptable level of about $100 \mathrm{C}^{\circ}$ [4]. Possible

\footnotetext{
* Work supported by US DoE

\# Permanent address: Budker INP, Novosibirsk 630090, Russia
}

progress in increasing the accelerating gradient will make pulsed heating even more serious.

The structure life-time drops exponentially when the temperature rise increases; [1,2] thus in order to make reliable measurements within a reasonable time one has to have a high temperature rise, up to $500-600{ }^{\circ} \mathrm{C}$. Tests at $34 \mathrm{GHz}$ can produce such temperature rises. The asymptotic behavior of the surface degradation may be determined based on measurements for different temperature rises that will allow firm conclusions to be drawn as to structure life-time.

In present paper a test device is described, to be driven with power from the $34.272 \mathrm{GHz}$ magnicon, [5] to determine the maximum achievable surface field limit imposed by pulsed heating.

\section{TEST CAVITY DESIGN}

The $\mathrm{TE}_{011}$ test cavity schematic layout and the rf magnetic field pattern are shown in Fig. 1. The cavity consists of two main parts, the cavity body which is coupled to an input waveguide, and the removable end cap, the left-most element in Fig. 1.

The cavity design has to satisfy two main requirements: (a) the cavity geometry including input coupling must be axially symmetric in order to keep surface electric fields negligibly small, and consequently to avoid breakdown problems, and (b) in order not to damage the main part of the cavity surface, the temperature rise at any point (except for the end cap) should not exceed the "safe" value of $110^{\circ} \mathrm{C}$. Because the end cap has to be tested up to $500{ }^{\circ} \mathrm{C}$, the maximum current density on its tip must be at least $2-2.5$ times higher than at any other point on the cavity surface.

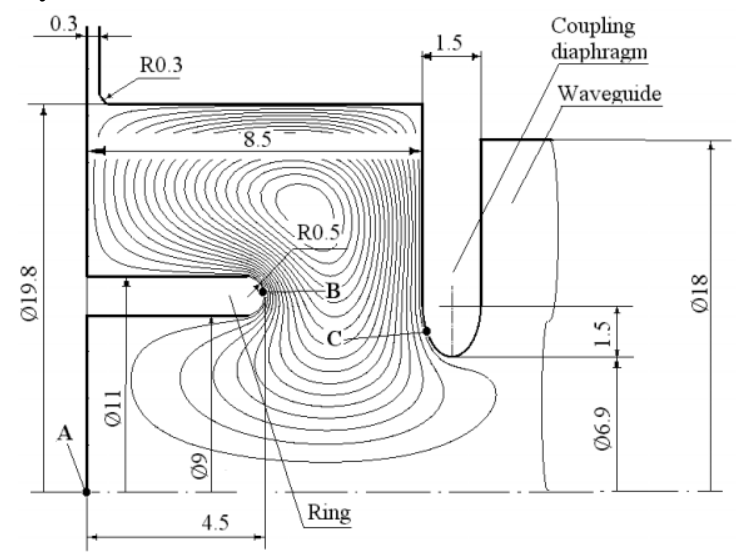

Fig. 1. The cavity schematic layout and the rf magnetic field pattern. All dimensions are in $\mathrm{mm}$.

The test cavity geometry shown in Fig. 1 has been developed in order to satisfy both requirements. In Fig 1. 
one can see that the input coupling diaphragm is elliptical in cross section with an eccentricity (i.e., ratio of the axial-to-radial semi-axes.) of 0.5 , in order to reduce surface current density at the iris edge. The end cap has a ring with diameter of $\sim 1 \mathrm{~cm}$ to achieve the required enhancement in surface current density (e.g., RF magnetic field). The RF magnetic field distribution along the cavity surface and the corresponding temperature rise are shown in Fig. 2.

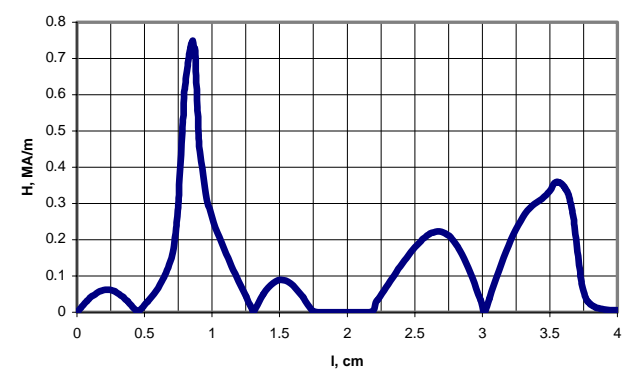

a)

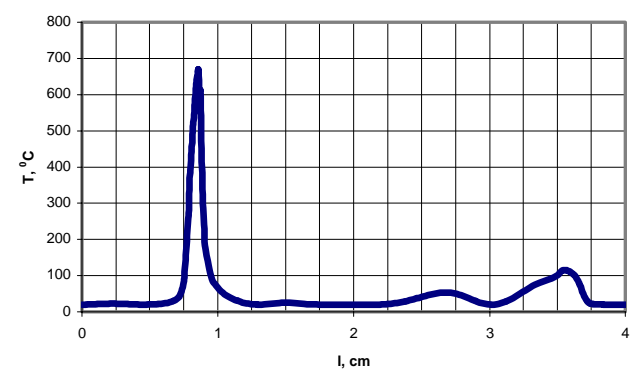

b)

Fig. 2. The magnetic field ( $a$ ) and temperature rise $(b)$ distributions over the cavity surface. Input power is 1.5 MW; pulse width is $1 \mu$ sec.

The axial point $l=0$ is the center of end cap (point $A$ in Fig. 1). The highest maximum surface magnetic field is at point $B$ on the ring, and the last maximum (at $l$ of about $35 \mathrm{~mm}$ ) is at point $C$ at the coupling iris. One can see that the magnetic field near the ring is at least two times greater than anywhere else in the cavity. The temperature distribution in Fig. $2 \mathrm{~b}$ is given for copper taking into account the increase in copper resistivity with temperature [6].

In Fig. 3. the maximum surface temperature of this cavity $v s$. the input power for a pulse width of $1 \mu \mathrm{sec}$ is presented. The lower curve corresponds to calculations without taking into account temperature dependence of the copper resistivity. The upper curve corresponds to calculations taking into account the cavity surface resistivity change during the pulse. The incident power reflection due to a mismatch, caused by decrease in the cavity quality factor is also taken into account. Note that this effect can be used as a technique for direct measurement of the temperature rise, which is difficult to do any other way.

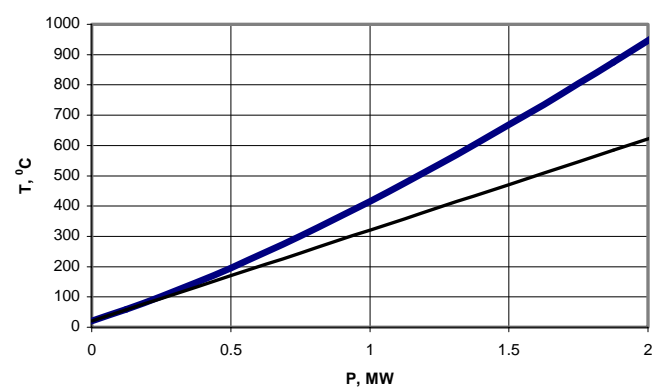

Fig. 3. Maximum surface temperature vs. input power for an initial temperature of $20^{\circ} \mathrm{C}$. See text for details.

The design parameters of the test cavity are listed in Table I.

Table I. Cavity parameters.

\begin{tabular}{|l||l|}
\hline operating frequency & $34.272 \mathrm{GHz}$ \\
\hline operating mode & $\mathrm{TE}_{011}$ \\
\hline quality factor (unloaded) & 7000 \\
\hline input power & $1.5 \mathrm{MW}$ \\
\hline maximum surface current density & $0.82 \mathrm{MA} / \mathrm{m}$ \\
\hline RF pulse duration & $1 \mu \mathrm{sec}$ \\
\hline temperature rise & $650^{\circ} \mathrm{C}$ \\
\hline input waveguide mode & $\mathrm{TE}_{01}$ \\
\hline
\end{tabular}

One can see that in order to achieve a temperature rise of $650{ }^{\circ} \mathrm{C}$ on the cavity surface during a $1 \mu$ sec rf pulse at $34.272 \mathrm{GHz}$, an rf power level of 1.5 MW is sufficient.

The test cavity mechanical design is shown in Fig. 4.

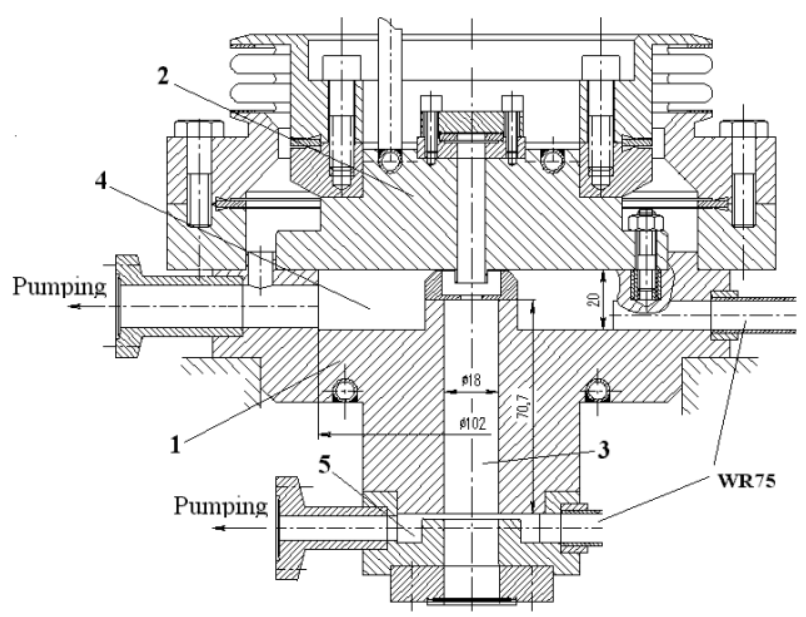

Fig. 4. Test cavity design. 1- cavity body; 2- end cap; 3- input waveguide; 4- coaxial chamber; 5- measuring chamber

The cavity is constituted of two main parts: cavity body (1) with coupling iris, and end cap (2). The end cap is separated from the cavity body by a gap, which allows 
one to replace the end cup, pump out the cavity and provide the possibility of microwave diagnostics. The end cap (2) is water cooled, which allows one to maintain constant temperature at before the pulse starts by regulating the water flow. One of the important parts of the experiment is the means for diagnosis of surface degradation due to metal fatigue. The major criterion that allows an indication of the state of the cavity surface is the $Q$-factor. It is necessary to make periodic measurements of the $Q$-factor without disassembling the cavity, so as to maintain high vacuum conditions. A hybrid dipole mode is used for this diagnosis. This mode has a resonant frequency of about $14 \mathrm{GHz}$. A substantial fraction of its Ohmic loss ( 20\%) is at the end of the field enhancement ring, which makes this mode sensitive to small changes in the damaged material conductivity. The mode is coupled to both coaxial chamber (4) and input waveguide (3). For this measurement the cavity can be excited through one WR-75 waveguide and measured through a second one (see Fig. 4). The test cavity input waveguide is not cut-off for the $14 \mathrm{GHz}$ dipole mode, and in order to prevent propagation of the test signal back to the input mode converter, the "measuring chamber" (5) is designed to constitute a choke for $14 \mathrm{GHz}$.

The schematic arrangement for pulsed heating experiment is shown in Fig. 5. All the magnicon ports are to have directional couplers. Because the minimum required $\mathrm{RF}$ power is about $2 \mathrm{MW}$, only one magnicon port will be used in the experiments. The three other ports will be connected to vacuum loads. The $\mathrm{TE}_{01}-\mathrm{TE}_{10}$ mode converter [7] is used to match the cylindrical waveguide in the $\mathrm{TE}_{01}$ mode with the WR28 rectangular waveguide at the magnicon output. A ceramic window separates the cavity from the magnicon in order to isolate high vacuum in the magnicon during the experiments with the cavity, e.g. when the cavity end cap is replaced.

Different materials can sustain different pulsed temperature rises, but the most important and common material for accelerating cavities is copper. Consequently, the first experiments will be done with copper. It is planned to perform experiments and assess damage to the cavity surface at different temperatures (from $500{ }^{\circ} \mathrm{C}$ to $200{ }^{\circ} \mathrm{C}$ ), which will allow one to extrapolate results to lower temperatures. After a certain number of pulses the end cap will be removed and investigated using facilities of the Yale Electron Microprobe Laboratory. Two major methods will be used for the end cap autopsy, Scanning Electron Microscopy (SEM), and Energy-Dispersive X-ray analysis (EDX), the latter to provide elemental concentration analysis.

\section{CONCLUSIONS}

At present the test cavity and the waveguide components are in construction. The $34 \mathrm{GHz}$ magnicon is undergoing initial tests [8]. Pulsed heating experiments are expected to begin within a year.

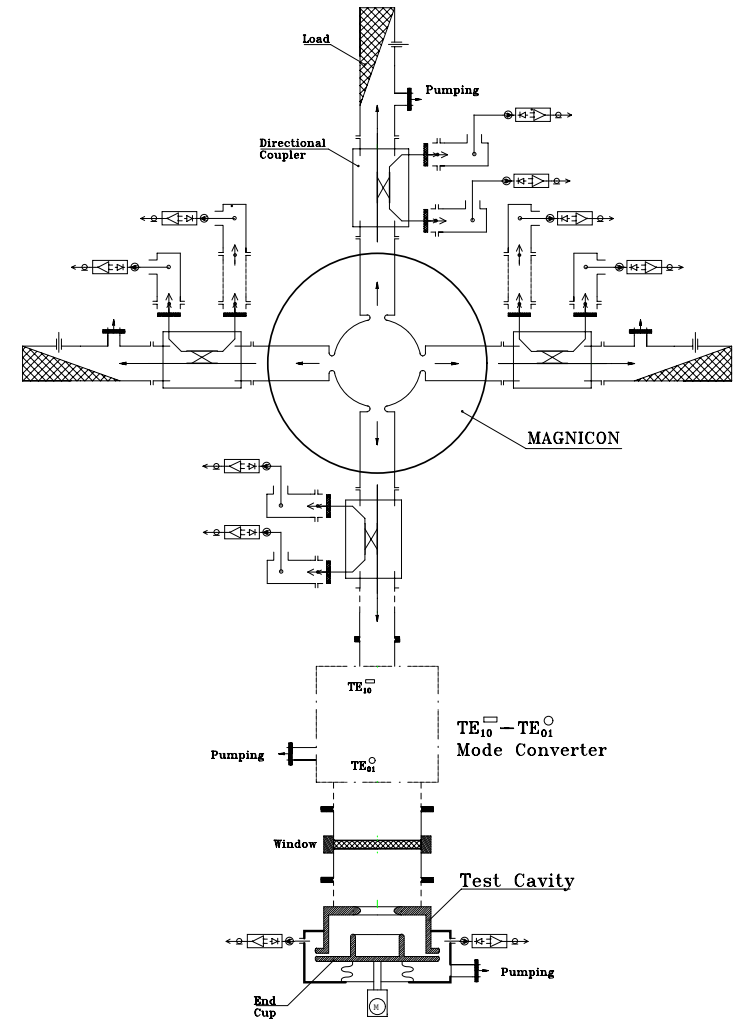

Fig. 5. Test bed layout $(14 \mathrm{GHz}$ diagnostics are not shown).

\section{REFERENCES}

[1] O.A. Nezhevenko, PAC97, Vancouver,1997, p.3013.

[2] V.F. Kovalenko, "Physics of Heat Transfer and Electrovacuum Devices", (Sovetskoe Radio, Moscow, 1975).

[3] D.P. Pritzkau and R.H. Sieman, Phys. Rev., v.5, 112002 (2002).

[4] J.W. Wang, et.al., LINAC2002, TH464.

[5] O.A. Nezhevenko, et al, AAC2002, AIP 647, p. 433 (2002).

[6] Handbook on Physics, I.K. Kikoin ed., Atomizdat, Moscow, 1976.

[7] G.G. Denisov, et al., AAC2002, AIP 647, p. 476 (2002).

[8] O.A. Nezhevenko, et al., "34 GHz, 45 MW Pulsed Magnicon: First Results," Present Conference, TPAE024. 